\title{
Pharmacokinetics of anidulafungin during venovenous extracorporeal membrane oxygenation
}

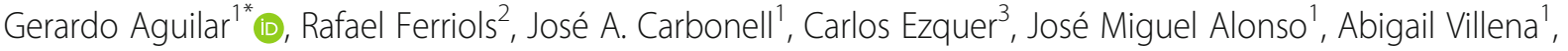 \\ Jaume Puig ${ }^{1}$, David Navarro ${ }^{4,5}$, Manuel Alós ${ }^{2}$ and F. Javier Belda ${ }^{1,5}$
}

Keywords: Echinocandins, Extracorporeal membrane oxygenation, Acute respiratory distress syndrome

Echinocandins are currently considered the first-line treatment for invasive candidiasis (IC) in the intensive care unit (ICU) $[1,2]$. However, extracorporeal membrane oxygenation (ECMO), a rescue therapy used in patients with severe acute respiratory distress syndrome (ARDS) [3], could alter the pharmacokinetics of certain drugs [4]. We prescribed anidulafungin for suspected IC in a patient with severe ARDS on ECMO and measured the plasma concentrations of the drug using highperformance liquid chromatography (HPLC).

A 69-year-old male patient was admitted to the ICU with septic shock secondary to peritonitis. The antiinfective treatment was based on surgical source control and broad spectrum antimicrobial therapy, including anidulafungin at usual doses. The patient developed severe ARDS. ECMO with Novalung iLA Activve ${ }^{\mathrm{Tx}}$ was initiated, maintaining ultraprotective ventilation. Femoral (23 F) and jugular (19 F) cannulas (Novalung ${ }^{\mathrm{Tm}}$, Germany) were inserted with $4.5 \mathrm{~L} / \mathrm{min}$ blood flow and $4 \mathrm{~L} / \mathrm{min}$ gas flow. Urine samples and pre-filter and postfilter blood samples were collected before starting the eight-dose anidulafungin infusion and $0.5,1,1.5,2,4,6$, 8 , and $24 \mathrm{~h}$ after the infusion ended. Anidulafungin was well tolerated without relevant adverse effects.
A non-compartmental pharmacokinetic analysis was performed using Abbottbase Pharmacokinetic Systems ${ }^{\mathrm{mm}}$ (Abbott Laboratories, Illinois, USA). The maximum and trough plasma concentrations $\left(C_{\max }\right.$ and $C_{\min }$, respectively) were estimated directly from concentration-time data. The area under the plasma concentration-time curve over the 24-h dosing interval $\left(\mathrm{AUC}_{0-24}\right)$ was estimated using the linear trapezoidal rule for both prefilter and post-filter data. Clearance $(\mathrm{CL})$ was estimated as dose/AUC ${ }_{0-24}$. The apparent volume of distribution at steady state $\left(\mathrm{V}_{\mathrm{ss}}\right)$ was estimated as the product of $\mathrm{CL}$ and mean residence time (MRT).

$\mathrm{C}_{\max }$ and $\mathrm{C}_{\min }$ were 13.5 and $2.19 \mathrm{mg} / \mathrm{L}$, respectively (Fig. 1). Pre-filter and post-filter $\mathrm{AUC}_{0-24}$ were 107 and $111 \mathrm{mg} / \mathrm{h} / \mathrm{L}$, respectively; $\mathrm{V}_{\mathrm{ss}}$ was $18.9 \mathrm{~L} ; \mathrm{CL}$ was $0.933 \mathrm{~L} / \mathrm{h}$. Urine anidulafungin concentrations were negligible. All pharmacokinetic data were comparable to published data in critically ill patients with and without other types of extracorporeal support [5].

Regarding the use of anti-infective drugs in patients on ECMO, most pharmacokinetic data on this topic are from neonatal studies of antibiotics [4]. To the best of our knowledge, this report is the first on the pharmacokinetics of anidulafungin in a critically ill patient on ECMO. In our case, the therapy had little effect on the pharmacokinetics, suggesting that the dose of anidulafungin does not need adjustment. However, future studies are needed to confirm these findings.

\footnotetext{
* Correspondence: gerardo.aguilar@uv.es

${ }^{1}$ Department of Anesthesiology and Intensive Care, Surgical Intensive Care Unit, Hospital Clínico Universitario de Valencia, Avenida Blasco Ibáñez, 17, 46010 Valencia, Spain

Full list of author information is available at the end of the article
} 


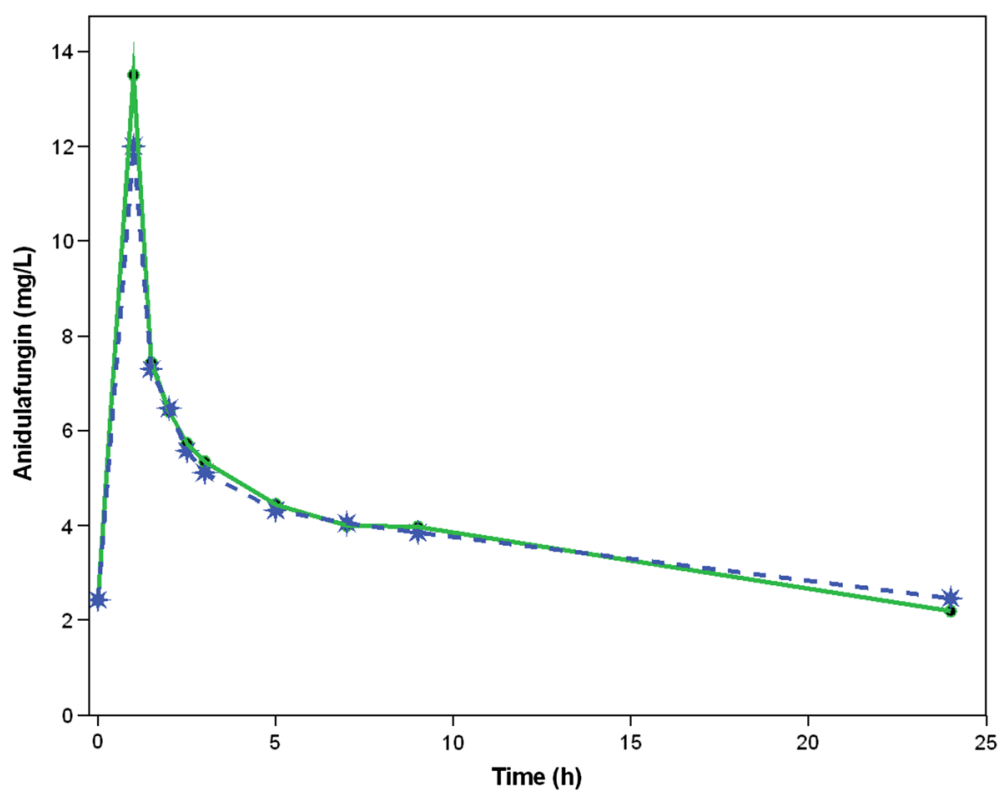

Fig. 1 Plasma anidulafungin concentrations over $24 \mathrm{~h}$. Solid and dotted lines represent the concentrations in the pre-filter and post-filter sides of the membrane, respectively. Urine concentrations of anidulafungin were very low and close to the limit of detection for the analytical procedure used $(0.05 \mathrm{mg} / \mathrm{L})$

\section{Abbreviations}

ARDS: Acute respiratory distress syndrome; $\mathrm{AUC}_{0-24}$ : Area under the plasma concentration-time curve over the 24-h dosing interval: $\mathrm{CL}$ : Clearance; $C_{\max }$ : Maximum plasma concentration; $C_{\min }$ : Trough plasma concentration; ECMO: Extracorporeal membrane oxygenation; HPLC: High-performance liquid chromatography; IC: Invasive candidiasis; ICU: Intensive care unit; MRT: Mean residence time; $V_{\text {ss: }}$ Apparent volume of distribution at steady state

\section{Funding}

The authors received no specific funding for this work.

\section{Availability of data and materials}

All relevant data are within the paper and its supporting information files. All data are fully available without restriction.

\section{Authors' contributions}

GA conceived the study, participated in its design, and helped draft the manuscript. RF, CE, and MA participated in designing the study and carried out the pharmacokinetics study. JMA, JAC, AV, and JP participated in analyzing and interpreting the data. DN and FJB participated in designing and coordinating the study and helped draft the manuscript. All authors read and approved the final manuscript.

\section{Competing interests}

GA received funds for speaking at meetings organized on behalf of Astellas, Gilead, Merck Sharp and Dohme (MSD), and Pfizer, as well as unrestricted research grants from Astellas, MSD, and Pfizer. DN received funds for speaking at meetings organized on behalf of Astellas, MSD, and Pfizer and received unrestricted research grants from Astellas and Pfizer. All other authors declare that they have no competing interests.

\section{Ethics approval and consent to participate}

The study (ANI-ECMO-2016) was approved by the local ethics committee (INCLIVA, Institute of Research, Valencia, Spain) and written informed consent obtained from the patient's next of kin.

\section{Author details}

${ }^{1}$ Department of Anesthesiology and Intensive Care, Surgical Intensive Care Unit, Hospital Clínico Universitario de Valencia, Avenida Blasco Ibáñez, 17, 46010 Valencia, Spain. ²Department of Pharmacy, Hospital Clínico Universitario de Valencia, Avenida Blasco Ibáñez, 17, 46010 Valencia, Spain. ${ }^{3}$ INCLIVA, Institute of Research, Avenida Blasco Ibáñez, 17, 46010 Valencia, Spain. ${ }^{4}$ Department of Microbiology, Hospital Clínico Universitario de Valencia, Avenida Blasco Ibáñez, 17, 46010 Valencia, Spain. ${ }^{5}$ School of Medicine, University of Valencia, Avenida Blasco Ibáñez, 15, 46010 Valencia, Spain.

\section{Published online: 17 October 2016}

\section{References}

1. Cornely OA, Bassetti M, Calandra T, ESCMID Fungal Infection Study Group, et al. ESCMID guideline for the diagnosis and management of Candida diseases 2012: non-neutropenic adult patients. Clin Microbiol Infect. 2012;18(Suppl. 7):19-37.

2. Pappas PG, Kauffman CA, Andes DR, et al. Clinical Practice Guideline for the Management of Candidiasis: 2016 Update by the Infectious Diseases Society of America. Clin Infect Dis. 2016;62(4):e1-50.

3. Gattinoni L. Ultra-protective ventilation and hypoxemia. Crit Care. 2016:20(1):130.

4. Sherwin J, Heath T, Watt K. Pharmacokinetics and dosing of anti-infective drugs in patients on extracorporeal membrane oxygenation: a review of the current literature. Clin Ther. 2016. doi:10.1016/j.clinthera.2016.07.169

5. Liu P, Ruhnke M, Meersseman W, Paiva JA, Kantecki M, Damle B. Pharmacokinetics of anidulafungin in critically ill patients with candidemia/ invasive candidiasis. Antimicrob Agents Chemother. 2013;57(4):1672-6. 